\title{
Spin-1 Particle in the Light-Front Approach
}

\author{
J. P. B. C. de Melo ${ }^{a}$ and T. Frederico ${ }^{b}$ \\ ${ }^{a}$ Instituto de Física Teórica, Universidade Estadual Paulista, 01405-900, São Paulo, SP, Brazil \\ ${ }^{b}$ Departamento de Física, Instituto Tecnológico de Aeronáutica, Centro Técnico Aeroespacial, \\ 12.228-900, São José dos Campos, São Paulo, Brazil
}

Received on 7 October, 2003

\begin{abstract}
The electromagnetic current of spin-1 composite particles does not transform properly under rotations if only the valence contribution is considered in the light-front model. In particular, the plus component of the current, evaluated only for the valence component of the wave function, in the Drell-Yan frame violates rotational symmetry, which does not allow a unique calculation of the electromagnetic form-factors. The prescription suggested by Grach and Kondratyuk [Sov. J. Nucl. Phys. 38, 198 (1984)] to extract the form factors from the plus component of the current, eliminates contributions from pair diagrams or zero modes, which if not evaluated properly cause the violation of the rotational symmetry. We address this problem in an analytical and covariant model of a spin-1 composite particle.
\end{abstract}

\section{Introduction}

Light-front models are useful to describe hadronic bound states, like mesons or baryons due to its particular boost properties [1, 2]. However, the light-front description in a truncated Fock-space breaks the rotational symmetry because the associated transformation is a dynamical boost [3, $4,5]$. It is a formidable task to study the properties under dynamical boosts in light-front quantization [2]. Therefore, an analysis with covariant analytical models, can be useful to pin down the main missing features in a truncated light-front Fock-space description of the composite system. In this respect, the rotational symmetry breaking of the plus component of the electromagnetic current, in the Drell-Yan frame, was recently studied within an analytical model for the spin1 vertex of a composite two-fermion bound state [3, 4]. It was shown that, if pair term contributions are ignored in the evaluation of the matrix elements of the electromagnetic current, the covariance of the form factors is lost $[3,4,5,6]$. The complete restoration of covariance in the form factor calculation is found only when pair terms or zero modes contributions to the matrix elements of the current are considered $[4,5,7,8]$.

The extraction of the electromagnetic form-factors of a spin-1 composite particle from the microscopic matrix elements of the plus component of the current $\left(J^{+}=J^{0}+J^{3}\right)$ in the Drell-Yan frame (momentum transfer $q^{+}=q^{0}+q^{3}=$ 0 ), based only on the valence component of the wave function, presents ambiguities due to the lacking of the rotational invariance of the current model $[9,10]$. In the Breit-frame with momentum transfers along the transverse direction (the Drell-Yan condition is satisfied) the current $J^{+}$has four independent matrix elements, although only three form factors exist. Therefore, the matrix elements satisfies an identity, known as the angular condition [9], which is violated.

Several extraction schemes for evaluating the form- factors were proposed, and in particular we consider the suggestion made in Ref. [9]. It was found in a numerical calculation of the $\rho$-meson electromagnetic form factors considering only the valence contribution [3], that the prescription proposed by [9] to evaluate the form-factors, produced results in agreement with the covariant calculations. In Ref. [3], it was used an analytical form of the $\rho$-quarkantiquark vertex. Later, in Ref. [6], it was shown that the above prescription eliminates the pair diagram contributions to the form factors, using a simplified form of the model, when the matrix elements of the current were evaluated for spin-1 light-cone polarization states. This nice result was thought to be due to the use of the particular light-cone polarization states. Here, we will show in a straightforward and analytic manner that the cancellation of the pair contribution in the evaluation of the form factors using the prescription from Ref. [9] also happens for the instant form polarization states in the cartesian representation, generalizing the previous conclusion [6]. Our aim, is to expose in a simple and detailed form, how the pair terms appear in the matrix elements of the current evaluated between instant form polarization states, and their cancellation in the form factors using the correct prescription. Therefore, we conclude that this property is more general than realized before.

We should observe that, in the case of spin- 0 composite particles (like the pion) with the correspondent form of the analytical model, the plus component of the electromagnetic current in the Breit-frame, with $q^{+}=0$, does not have contributions from pair terms $[8,11]$. It is enough to evaluate the valence part of the matrix element of the current to reproduce the covariant result. We remind that in the case of other components of the current, like $J^{-}=J^{0}-J^{3}$, the pair term contributes to the matrix element of the current [8].

This work is organized as follows. In Sect. II, we present the light-front model of the spin-1 particle, and evaluate the matrix elements of the plus component of the electromag- 
netic current in the Breit-frame with $q^{+}=0$. We separate out the pair terms in the matrix elements using the pole dislocation method $[4,7,5,8]$. In Sect. III, we show the cancellation of the pairs terms when the form factors are evaluated with the instant form polarization states with the prescription suggested by Grach and Kondratyuk [9]. In Sect. IV, we present our conclusion.

\section{Light-front model}

The electromagnetic (e.m.) current has the following general form for spin-1 particles [12]:

$$
\begin{aligned}
& J_{\alpha \beta}^{\mu}=\left[F_{1}\left(q^{2}\right) g_{\alpha \beta}-F_{2}\left(q^{2}\right) \frac{q_{\alpha} q_{\beta}}{2 m_{\rho}^{2}}\right]\left(p^{\mu}+p^{\prime \mu}\right) \\
& -F_{3}\left(q^{2}\right)\left(q_{\alpha} g_{\beta}^{\mu}-q_{\beta} g_{\alpha}^{\mu}\right)
\end{aligned}
$$

where $m_{v}$ is the mass of the vector particle, $q^{\mu}$ is the momentum transfer, $p^{\mu}$ and $p^{\prime \mu}$ is on-shell initial and final momentum respectively. From the covariant form factors $F_{1}$, $F_{2}$ and $F_{3}$, one can obtain the charge $\left(G_{0}\right)$, magnetic $\left(G_{1}\right)$ and quadrupole $\left(G_{2}\right)$ form factors (see e.g. [3]).

In the impulse approximation for the elastic photoabsorption amplitude (represented by a Feynman triangle diagram), the matrix elements of the e.m. current, $J^{+}$is written as [3]:

$$
\begin{aligned}
& J_{j i}^{+}=\imath \int \frac{d^{4} k}{(2 \pi)^{4}} \frac{\operatorname{Tr}[]_{j i} \Lambda\left(k, p^{\prime}\right) \Lambda(k, p)}{\left((k-p)^{2}-m^{2}+\imath \epsilon\right)\left(k^{2}-m^{2}+\imath \epsilon\right)} \\
& \times \frac{1}{\left(\left(k-p^{\prime}\right)^{2}-m^{2}+\imath \epsilon\right)},
\end{aligned}
$$

where the trace is given by:

$$
\begin{aligned}
& \operatorname{Tr}[]_{j i}=\operatorname{Tr}\left[\epsilon_{j}^{\prime \alpha} \Gamma_{\alpha}\left(k, k-p^{\prime}\right)\left(k-p^{\prime}+m\right) \gamma^{+}\right. \\
& \left.\times(k-p+m) \epsilon_{i}^{\beta} \Gamma_{\beta}(k, k-p)(k+m)\right],
\end{aligned}
$$

with $\gamma^{+}=\gamma^{0}+\gamma^{3}$. The polarization four-vectors of the initial and final states are $\epsilon_{i}$ and $\epsilon_{j}^{\prime}$, respectively. The regularization function $\Lambda(k, p)=N /\left((p-k)^{2}-m_{R}^{2}+\imath \epsilon\right)$ is used to keep finite the photo-absorption amplitude. The regularization parameter is $m_{R}$.

In this work, we make use of a simple Dirac structure in the spinor space for the vertex $\Gamma_{\alpha}(k, k-p)$, which is written as $\Gamma=\Gamma^{\prime}=\gamma^{\mu}$. Although, this vertex has a simple structure, it originates $S$ and $D$ states in the relative motion of the fermions which composes the spin- 1 particle. The full form of the vertex, applied previously to study the $\rho$-meson e.m. form factors, has one more term which puts the quarks on the relative $S$ state [3]. With only the $\gamma^{\mu}$ structure, the trace of Eq. (3) becomes:

$\operatorname{Tr}[]_{j i}=\operatorname{Tr}\left[k_{f}^{\alpha}\left(k-\not p^{\prime}+m\right) \gamma^{+}(k-\not p+m) k_{i}^{\alpha}(k+m)\right]$.

Using the light-front coordinates as $k^{+}=k^{0}+k^{+}, k^{-}=$ $k^{0}-k^{3}$ and $\left.k_{\perp}=\left(k_{x}, k_{y}\right)\right)$, the $k^{-}$dependence in the trace is separated. Following [5], we treat the $k^{-}$factors which come from the instantaneous term of the fermion propagators carefully, because they can cause the violation of the rotational symmetry of the plus component of the e.m. current. These contributions are related to the presence of pair terms in the matrix elements of the e.m. current in the limit where $q^{+}=\delta^{+}$goes to zero [5].

Writing only the $k^{-}$dependence of the trace, one has:

$$
\operatorname{Tr}[]_{j i}^{B a d}=\frac{k^{-}}{2} \operatorname{Tr}\left[k_{j}^{\prime \alpha}\left(k-p^{\prime}+m\right) \gamma^{+}(k-p+m) k_{i}^{\alpha} \gamma^{+}\right]
$$

The terminology "Bad" is used here to indicate the possible contribution of pair terms.

The matrix elements of the e.m. current are calculated in the Breit frame with the momentum transfer $q^{\mu}=$ $\left(0, q_{x}, 0,0\right), p^{\mu}=\left(p^{0},-q_{x} / 2,0,0\right)$ for the spin-1 particle initial state and $p^{\prime \mu}=\left(p^{0}, q_{x} / 2,0,0\right)$ for the final state. Introducing the useful definition $\eta=q^{2} / 4 m_{v}$, we have $p^{0}=m_{v} \sqrt{1+\eta}$. The instant-form polarization four-vectors in the cartesian representation are given by $\epsilon_{x}^{\mu}=(-\sqrt{\eta}, \sqrt{1+\eta}, 0,0), \epsilon_{y}^{\mu}=\epsilon_{y}^{\prime \mu}=(0,0,1,0)$, $\epsilon_{z}^{\mu}=\epsilon_{z}^{\prime \mu}(0,0,0,1)$ and $\epsilon_{x}^{\prime \mu}=(\sqrt{\eta}, \sqrt{1+\eta}, 0,0)$. For these polarization four-vectors, the traces are given by:

$$
\begin{aligned}
& \operatorname{Tr}[]_{x x}^{\text {Bad }}=k^{-\frac{\eta}{8}} R, \\
& \operatorname{Tr}[]_{y y}^{\text {Bad }}=k^{-}\left(k^{+}-p^{+}\right)^{2}, \\
& \operatorname{Tr}[]_{z z}^{\text {Bad }}=\frac{1}{8} k^{-} R, \\
& \operatorname{Tr}[]_{z x}^{\text {Bad }}=-k^{-\frac{\sqrt{\eta}}{8}} R,
\end{aligned}
$$

where

$$
R=4 \operatorname{Tr}\left[\left(k-\not p^{\prime}+m\right) \gamma^{+}(k-\not p+m) \gamma^{-}\right] .
$$

The next step is to evaluate matrix elements of the electromagnetic current for the "Bad" traces calculated above in Eq. (7). To perform the integration of the light-front energy, $k^{-}$, in Eq. (2) one needs to use the pole dislocation method, developed in Refs. [4, 5, 7, 8], where it is used $q^{+}=\delta^{+} \rightarrow 0_{+}$. In detail, the pair terms or Z-diagram contributions are given by:

$$
\begin{aligned}
J_{x x}^{+Z} & =\lim _{\delta^{+} \rightarrow 0} \int d^{4} k \frac{\theta\left(p^{+}-k^{+}\right) \theta\left(k^{+}-p^{+}\right) \operatorname{Tr}[]_{x x}^{\text {Bad }}}{[1][2][3][4][5][6][7]} \\
J_{y y}^{+Z} & =\lim _{\delta^{+} \rightarrow 0} \int d^{4} k \frac{\theta\left(p^{\prime+}-k^{+}\right) \theta\left(k^{+}-p^{+}\right) \operatorname{Tr}[]_{y y}^{\text {Bad }}}{[1][2][3][4][5][6][7]} \\
J_{z x}^{+Z} & =\lim _{\delta^{+} \rightarrow 0} \int d^{4} k \frac{\left.\theta\left(p^{\prime+}-k^{+}\right) \theta\left(k^{+}-p^{+}\right) \operatorname{Tr}[]_{z x}^{\text {Bad }}\right]}{[1][2][3][4][5][6][7]} \\
J_{z z}^{+Z} & =\lim _{\delta^{+} \rightarrow 0} \int d^{4} k \frac{\theta\left(p^{\prime+}-k^{+}\right) \theta\left(k^{+}-p^{+}\right) \operatorname{Tr}[]_{z z}^{\text {Bad }}}{[1][2][3][4][5][6][7]}
\end{aligned}
$$

(4) The results shown above correspond only to the computation of the $k^{-}$integration for $p^{+}<k^{+}<p^{\prime+}$ with $p^{\prime+}=p^{+}+\delta^{+}$, where the pair term contribution to the plus component of the electromagnetic current appears $[4,5]$. The terms of the form $k^{-(m+1)}\left(p^{+}-k^{+}\right)^{n}$ in Eq. (8) are zero in the limit $\delta \rightarrow 0_{+}$if $m<n$ [5]. Therefore, one immediately get that $J_{y y}^{+Z}=0$, which does not have a pair 
term contribution, as has been already verified explicitly in $[3,5]$.

The denominators in Eq. (8), written with light-front momentum are given by:

$$
\begin{aligned}
& {[1]=k^{+}\left(k^{-}-\frac{f_{1}-\imath \epsilon}{k^{+}}\right) ;} \\
& {[2]=\left(p^{+}-k^{+}\right)\left(p^{-}-k^{-}-\frac{f_{2}-\imath \epsilon}{p^{+}-k^{+}}\right) ;} \\
& {[3]=\left(p^{+}-k^{+}\right)\left(p^{\prime-}-k^{-}-\frac{f_{3}-\imath \epsilon}{p^{+}-k^{+}}\right) ;} \\
& {[4]=\left(p^{+}-k^{+}\right)\left(p^{-}-k^{-}-\frac{f_{4}-\imath \epsilon}{p^{+}-k^{+}}\right) ;} \\
& {[5]=\left(p^{+}-k^{+}\right)\left(p^{-}-k^{-}-\frac{f_{5}-\imath \epsilon}{p^{\prime+}-k^{+}}\right) ;} \\
& {[6]=\left(p^{+}-k^{+}\right)\left(p^{-}-k^{-}-\frac{f_{6}-\imath \epsilon}{p^{\prime+}-k^{+}}\right) ;} \\
& {[7]=\left(p^{+}-k^{+}\right)\left(p^{\prime-}-k^{-}-\frac{f_{7}-\imath \epsilon}{p^{++}-k^{+}}\right) ;}
\end{aligned}
$$

where the functions $f_{i}$ are

$$
\begin{aligned}
& f_{1}=k_{\perp}^{2}+m^{2} ; \\
& f_{2}=(k-p)_{\perp}^{2}+m^{2} ; \\
& f_{3}=\left(k-p^{\prime}\right)_{\perp}^{2}+m^{2} ; \\
& f_{4}=(k-p)_{\perp}^{2}+m_{R}^{2} ; \\
& f_{5}=\left(k-p^{\prime}\right)_{\perp}^{2}+m_{R}^{2} ; \\
& f_{6}=(k-p)_{\perp}^{2}+m_{R}^{2} ; \\
& f_{7}=\left(k-p^{\prime}\right)_{\perp}^{2}+m_{R}^{2} .
\end{aligned}
$$

A final comment is appropriate here, the interval $0<$ $k^{+}<p^{+}$in the integration of Eq. (8) implies in a nonvanishing result of the $k^{-}$integration, where the residue receives contribution from the pole at $k^{-}=\left(f_{1}-\imath \epsilon\right) / k^{+}$. This result is part of the valence contribution to the electromagnetic current.

\section{Form factors and pair terms}

The angular condition $[3,9,10]$ satisfied by the matrix elements (m.e.) of $J^{+}$in the Breit-frame with $q^{+}=0$, due to the requirements of rotational symmetry and parity conservation is written as

$$
\Delta\left(q^{2}\right)=(1+2 \eta) I_{11}^{+}+I_{1-1}^{+}-\sqrt{8 \eta} I_{10}^{+}-I_{00}^{+}=0,
$$

with the m.e. of the plus component of the current evaluated the light-cone polarization states, denoted as $I_{m^{\prime} m}^{+}$. Different prescriptions to extract the form factors choose three m.e. among the four independent ones (if only the valence contribution is evaluated), or any other three linearly independent combinations of matrix elements. The angular condition in the instant form spin basis takes a particularly simple form

$$
J_{y y}^{+}=J_{z z}^{+} \text {. }
$$

The prescription suggested by Grach and Kondratyuk [9] eliminates the matrix element $I_{00}^{+}$from the computation of the form factors using the angular condition.
The electromagnetic form factors are linear combinations of the matrix elements of the electromagnetic current, and using the prescription [9], one has

$$
\begin{aligned}
G_{0}^{G K} & =\frac{1}{3}\left[J_{x x}^{+}+J_{y y}^{+}(2-\eta)+\eta J_{z z}^{+}\right] ; \\
G_{1}^{G K} & =\left[J_{y y}-J_{z z}^{+}-\frac{J_{z x}^{+}}{\eta}\right] ; \\
G_{2}^{G K} & =\frac{\sqrt{2}}{3}\left[J_{x x}^{+}+J_{y y}^{+}(-1-\eta)+\eta J_{z z}^{+}\right] ;
\end{aligned}
$$

where the transformation of the light-cone to the instant form polarization states were performed.

Now, due to the property of the traces of Eq. (6), i.e.,

$$
\begin{aligned}
& \operatorname{Tr}[]_{x x}^{B a d}=-\eta \operatorname{Tr}[]_{z z}^{B a d} ; \\
& \operatorname{Tr}[]_{z x}^{B a d}=-\sqrt{\eta} \operatorname{Tr}[]_{z z}^{\text {Bad }} ;
\end{aligned}
$$

one immediately derives from Eq. (8) that

$$
\begin{aligned}
& J_{x x}^{+Z}=-\eta J_{z z}^{+Z} \\
& J_{z x}^{+Z}=-\sqrt{\eta} J_{z z}^{+Z} .
\end{aligned}
$$

Substituting these matrix elements in Eq. (13), we compute the contribution of the pair terms to the form factors:

$$
\begin{aligned}
G_{0}^{G K, Z} & =\frac{1}{3}\left[J_{x x}^{+Z}+\eta J_{z z}^{+Z}\right]=0 ; \\
G_{1}^{G K, Z} & =\left[-J_{z z}^{+Z}-\frac{J_{z x}^{+Z}}{\eta}\right]=0 ; \\
G_{2}^{G K, Z} & =\frac{\sqrt{2}}{3}\left[J_{x x}^{+Z}+\eta J_{z z}^{+Z}\right]=0 ;
\end{aligned}
$$

where we have also used that $J_{y y}^{+Z}=0$.

The vanishing of the contribution of the pair terms as seen in Eqs. (16), explains part of the results obtained by the authors in Ref. [3], where it was found by numerical analysis, that the prescription of Grach and Kondratyuk with only the valence contribution reproduced the covariant calculation.

\section{Conclusion}

In summary, we have analyzed the rotational symmetry properties of the electromagnetic current in an analytical light-front model of a spin- 1 composite particle. The plus component of the current in the Drell-Yan frame does not transform properly under rotations if only the valence contribution is considered in the model. This problem forbids a unique calculation of the electromagnetic form-factors, due to the violation of the angular condition.

We have analyzed in deep, a particular prescription suggested by Grach and Kondratyuk to extract the form factors from the plus component of the current, and we have shown that it eliminates the contributions from pair diagrams or zero modes, which if not evaluated properly cause the violation of the rotational symmetry. We have addressed this 
problem in an analytical and covariant model of a spin-1 composite particle using a a pole dislocation method to calculate the pair terms which survive the limit of $q^{+} \rightarrow 0_{+}$. We have shown, in a direct and compact form, the cancellation of the pair contribution in the evaluation of the form factors using the prescription from Ref. [9], which also happens using the instant form polarization states in the cartesian representation, generalizing previous findings [6]. In a future work, we intend to analyze with our methods the above prescription, to extract form factors from the electromagnetic current, with a more general form of the coupling of the composite vector particle to the fermions. The goal is to apply the results of such analysis to the study of the $\rho$-meson or deuteron elastic photo-absorption processes.

\section{Acknowledgments}

This work was supported in part by the Brazilian agencies FAPESP (Fundação de Amparo a Pesquisa do Estado de São Paulo) and CNPq (Conselho Nacional de Desenvolvimento Ciêntifico e Técnologico).

\section{References}

[1] M.V. Terentév, Sov. J. Nucl. Phys. 24, 106 (1976); L.A. Kondratyuk and M.V. Terentév, Sov. J. Nucl. Phys. 31, 561
(1980).

[2] S. J. Brodsky, H.-C. Pauli, and S. S. Pinsky, Phys. Rep. 301, 299 (1998).

[3] J.P.B.C. de Melo and T. Frederico, Phys. Rev. C55, 2043 (1997).

[4] J.P.B.C. de Melo, J.H.O.Sales, T. Frederico, and P.U. Sauer, Nucl. Phys. A631, 574c (1998).

[5] J.P.B.C. de Melo, T. Frederico, H.W.L. Naus, and P.U. Sauer, Nucl. Phys. A660, 219 (1999).

[6] B.L.G. Bakker and C.R. Ji, Phys. Rev. D65, 116001 (2002).

[7] H.W.L. Naus, J.P.B.C. de Melo, and T. Frederico, Few-Body Systems 24, 99 (1998).

[8] J.P.B.C. de Melo, H.W. L. Naus, and T. Frederico, Phys. Rev. C59, 2278 (1999).

[9] I.L. Grach and L.A. Kondratyuk, Sov. J. Nucl. Phys. 38, 198 (1984).

[10] I.L. Grach, L.A. Kondratyuk, and M. Strikman, Phys. Rev. Lett. 62, 387 (1989).

[11] J.P.B.C. de Melo, T. Frederico, E. Pace, and G. Salmé, Nucl. Phys. A707, 399 (2002); Braz. J. Phys. 33, 301 (2003).

[12] L.L. Frankfurt and M. Strikman, Nucl. Phys. B148, 107 (1979); Phys. Rep. 76, 215 (1981). 